\title{
CORRIGENDUM
}

\section{The relationship between anxiety, depression and religious coping strategies and erectile dysfunction in Iranian patients with spinal cord injury}

AH Pakpour, P Rahnama, H Saberi, M Saffari, V Rahimi-movaghar, A Burri and M Hajiaghababaei

Spinal Cord (2017) 55, 711; doi:10.1038/sc.2017.32

Correction to: Spinal Cord (2016) 54, 1053-1057; doi:10.1038/ sc.2016.7; published online 16 February 2016

Since the publication of this article, the authors have noticed that the affiliation for author M Hajiaghababaei was incorrect. The correct affiliation for this author is:
${ }^{3}$ Brain and Spinal Cord Injury Research Center, Neuroscience Institute, Tehran University of Medical Sciences, Tehran, Iran

The authors apologise for any inconvenience caused. 